\title{
FACTORS INFLUENCING ACHIEVEMENT OF LOW-DENSITY LIPOPROTEIN CHOLESTEROL GOALS IN MEXICO: THE INTERNATIONAL ChOlesterol MANAGEMENT PRACTICE STUẸY
}

\author{
Omar Y. Bello-Chavolla ${ }^{1,2}$ and Carlos A. Aguilar-Salinas $1,3,4$
}

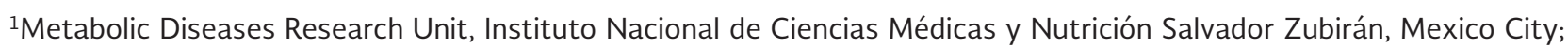
${ }^{2}$ Combined Studies Program in Medicine MD/PhD (PECEM), Faculty of Medicine, Universidad Nacional Autónomą de México, Mexico City; ${ }^{3}$ Department of Endocrinology and Metabolism, Instituto Nacional de Ciencias Médicas ${ }^{\circ}$ y Nutrición Salvador Zubirán, Mexico City; ${ }^{4}$ School of Medicine and Health Sciences, Tecnológico de Monterrey, $\frac{C}{(1)}$ Monterrey, Mexico

\begin{abstract}
\end{abstract}
Background: The International Cholesterol Management Practice Study is a multinational collaborative effort to describe the effectiveness of the lipid-lowering therapy (LLT) as well as the main barriers to achieve the low-density lipoprotein chotesterol (LDL-C) goals. Objective: The objective of the study was to investigate factors associated with the achievement of LDL $=C$ goals in Mexico using real-life data. Methods: This was a cross-sectional observational study from 18 physicians acros\$ different health facilities in Mexico, who provided information about their practices between August 2015 and August $2016=\mathrm{We}$ included patients treated for $\geq 3$ months with any LLT in whom LDL-C measurement on stable LLT was available for the pifevious 12 months. Results: We included 623 patients with a mean age of $59.3 \pm 12.7$ years; $55.6 \%$ were women. The mean $L D L-C$ value on LLT was $141.8 \pm 56.1 \mathrm{mg} / \mathrm{dL}$. At enrollment, $97.4 \%$ of patients were receiving statin therapy ( $11.3 \%$ on high-intensity treatment). Only $24.8 \%$ of the very-high cardiovascular (CV) risk patients versus $26.4 \%$ of the high risk and $52.4 \%$ of the moderate risk patients achieved their LDL-C goals. Independent factors associated with non-achievement of LDL-C goal were statin intolerance, overweight and obesity, abdominal obesity, female sex, high CV risk, use of public health-care service, metabolic syndrome, type 2 diabetes, and hypertriglyceridemia. Higher-level of education was associated with a lower risk $\mathrm{gf}$ not achieving LDL-C goals. Conclusions: Achievement of LDL-C goals is suboptimal in Mexico, especially in patients with the highest CV risk. The main barriers to achieve the goal are easily detectable. Implementation of LLT should be adapted to the patient's needs and profile. (REV INVEST CLIN. 2019;71:408-16)

Key words: Cholesterol. low-density lipoprotein cholesterol. statins. lipids, Mexican population.

*Corresponding author:

Carlos A. Aguilar-Salinas

E-mail: caguilarsalinas@yahoo.com
Received for publication: 21-06-2019

Approved for publication: 07-08-2019

DOI: $10.24875 / R I C .19003156$ 


\section{INTRODUCTION}

High low-density lipoprotein-cholesterol (LDL-C) level is among the most common forms of dyslipidemia in Mexicans, being present in nearly half of the adult population. This strong risk factor for cardiovascular (CV) events remains largely untreated and underdiagnosed ${ }^{1}$. Management of dyslipidemia is centered in lowering the concentration of atherogenic particles, estimated with LDL-C levels. Lipid-lowering therapy (LLT) results in CV benefits and reduced rates of both $\mathrm{CV}$ events and mortality ${ }^{2}$. Statins are the first drug of choice, as recommended by most guidelines ${ }^{3-5}$. Despite the proven benefits of LLT, under treatment is a major area of concern in the management of dyslipidemia. Additional LLTs include ezetimibe and proprotein convertase subtilisin/ kexin type 9 (PCSK9) inhibitors, which have been shown to provide a clinical benefit in combination therapies, but their use is limited and often not considered in clinical practice by attending physicians.

Undertreatment is a multifactorial challenge, in which conditions related to patients, physicians, and health services mutually interact. Some of these factors could be overcome by the implementation of patient-centered strategies or with educational programs for physicians. Most studies reporting these factors have been conducted in Western Europe and North America ${ }^{6,7}$. The International ChoLesterol management Practice Study (ICLPS) was designed to provide the corresponding data in countries outside of Western Europe, areas in which growing trends in CV mortality have been reported during the past decade. Physician practices of 18 countries, including Mexico, were evaluated to measure achievement of LDL-C targets in patients who were already receiving stable LLT to identify factors independently linked to undertreatment. Here, we aim to provide an in-depth characterization of ICPLS data obtained from Mexican patients. This is, to our best knowledge, the first evaluation specifically dedicated to the identification of factors related to LDL-C goal nonachievement in our population.

\section{METHODS}

\section{Study Population and Patient Selection}

This report contains data collected for the ICLPS in Mexican participants ${ }^{8}$. ICLPS is a cross-sectional observational study which included adult patients treated in Mexican facilities who had been receiving a stable type and dose of LLT for $\geq 3$ months before enrollment and had their LDL-C value measured while receiving stable LLT in the previous 12 months. Patients participating in a clinical trial or who had re-

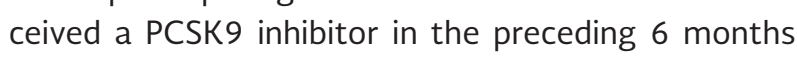
were excluded from the study. To ensure that the results adequately reflected the management $\mathrm{Of}$ dyslipidemic patients in real-life practice, the contribution of each medical specialty made in the management of such patients was provided by a national expert. To limit bias in the selection of the study sites; potential centers/physicians were identified because of their high demand of services, included in a list and selected using a randomization process contrôlled to ensure a balanced representation of each specialty. To limit patient selection bias, sites were instructed to recruit a minimum of five consecutive patients per site. A predefined 2-week interval was selected-during which all consecutive consenting patients who attended the visit for any reason were enrolled. As not all sites could start recruitment at the same fime, a timeframe of 3-6 months was given for recruitment depending on the total number of sites/patients.

\section{Data collection}

Physicians completed a questionnaire that coflected both patient and physician information. Fromphysicians, we obtained demographic data, medical specialty, years of practice, type and location of practice, main workplace, mean number of patients consulted per day, choice of and adherence to practicegguidelines for lipid disorders (i.e., European Society of Cardiology and European Atherosclerosis Societȳ ESC/ EAS, American College of Cardiology/Americam Heart Association [ACC/AHA], and other international/lo$\mathrm{cal} /$ national guidelines), and the definition that he or she used to diagnose statin intolerance (i.e, , $\underline{\underline{m}}$ tolerance to 1,2 , or $\geq 3$ statins). A case report form was completed for each patient during a single visist. The data collected included: demographic information; results of physical examination, CV risk factors, type of hypercholesterolemia (primary, secondary, or unknown), LDL-C values (calculated or measured directly; on current treatment and untreated if available) and other lipid variables, current use of LLTs and/or antithrombotic drugs, socioeconomic profile, and the investigator's assessment of the patient's CV risk 
level. Data quality control was performed by trained personnel at more than $10 \%$ of randomly chosen sites. Risk factors were defined as proposed by 2011 ESC/EAS guidelines; familial hypercholesterolemia was defined according to the Dutch Lipid Clinic Network $^{9}$.

\section{Statistical Analysis}

Baseline characteristics are presented using mean $( \pm S D)$ or median (interquartile range) values for continuous variables and as frequencies for categorical data. The primary outcome measure for this study was the proportion of patients taking LLT who did not achieve LDL-C targets as defined by 2011 ESC/EAS guidelines: $<1.8 \mathrm{mmol} / \mathrm{L}(70 \mathrm{mg} / \mathrm{dL})$ for very-high risk, $<2.5 \mathrm{mmol} / \mathrm{L}(100 \mathrm{mg} / \mathrm{dL})$ for high risk, and $<3.0 \mathrm{mmol} / \mathrm{L}(115 \mathrm{mg} / \mathrm{dL})$ for moderate-risk patients. The Systematic Coronary Risk Estimation (SCORE) chart for high-risk countries was used to stratify patients by their $\mathrm{CV}$ risk ${ }^{10}$. A series of logistic regression models were developed to test the relationship between non-achievement of LDL-C targets and demographic, clinical, and treatment characteristics. All analyses were conducted using SAS version 9.2.

\section{RESULTS}

\section{Physician Characteristics}

We included patients recruited by 18 physicians across Mexico ( $71.2 \%$ men, mean age of $48.3 \pm 10.9$ years, and $21.9 \pm 9.9$ years of practice) including nine general practitioners, two cardiologists, three internal medicine specialists, three endocrinologists, and one lipidologist. Most attended patients were from urban regions ( $94.4 \%$ ) and private practice settings (61.1\%). All but two physicians reported following specific guidelines or recommendations for the management of lipid disorders; the majority reported following the ACC/AHA Guidelines on the treatment of blood cholesterol ( $56.3 \%$ ), followed by the ESC/EAS Guidelines for the management of dyslipidemia (43.8\%). In general, physicians reported seeing a median of 5.0 (3.07.0) patients with dyslipidemia and/or with lipidmodifying treatments per day, representing $40.6 \%$ (26.7-50.0\%) of their daily practice.

\section{Patients' characteristics and CV risk}

From a total of 653 patients who were screened, 23 were ineligible for inclusion in the study, ang d four had incomplete information. Our final study sample was composed of 626 patients. The mean alge of the participants was $59.3 \pm 12.7$ years; $55.6 \%$ were women; $60.1 \%$ had native Latin American ancestry; $572(91.4 \%)$ were evaluated in urban areas; and $444(70.9 \%)$ completed secondary education or higher. Hypertension was present in $367 \%$ cases (58.6\%) and $413(66.0 \%)$ reported not doing regular physical activity. Type 2 diabetes (T2D) was present in 367 patients (58.6\%) with a median of $9.0(4.0-16.0)$ years from diagnosis; 91 pàtients (24.9\%) reported having diabetes-related microvascular complications, and $56(15.3 \%)$ reported experiencing at least one episode of symptomatic hypoglycemia in their lifetime. Median body mass index was $28.8 \mathrm{~kg} / \mathrm{m}^{2}$ (26.2-32); close to $40 \%$ of the study subjects were obese. A total of $1 \frac{145}{4} \mathrm{pa}-$ tients were former smokers and $412(65.8 \%)$ were current smokers. Metabolic syndrome as defined according to Adult Treatment Panel -III critefita was present in 342 patients (54.7\%), and in 407 ( $65.2 \%)$ according to IDF criteria.

Overall, 90 patients ( $14.4 \%$ ) had documented côronary artery disease, defined as a previous acute coronary syndrome $(77 / 90,85.6 \%)$, previous percutaneous coronary intervention $(61 / 90,67.8 \%)$, or previous coronary artery bypass graft $(15 / 90,16.7 \%)$. Twentyfour familial hypercholesterolemia cases were included in the study. The median (interquartile range) time since a diagnosis of dyslipidemia was 4.0 (1.0-7.0) years. Of $498(79.6 \%)$ patients in whom the SCORE CV risk could be calculated, 210 (42.2\%) wereat very high risk, 235 (47.2\%) were at high risk, 42 \$8.4\%) were at moderate risk, and 11 (2.2\%) were at low risk.

Physician-estimated CV risk correlated poorly with the calculated risk. Over half of the patients az high/ very-high calculated risk were estimated by physicians to be at a lower-risk level (Fig. 1). Conversely, $47.2 \%$ of calculated low and moderate risk patients were estimated by physicians to be at a higher level of risk. The LDL-C value at the time of the first diagnosis before starting LLT was available in 175 (27.9\%) patients. The mean value was $141.8 \pm 56.1 \% \mathrm{mg} / \mathrm{dL}$ ( $3.7 \pm 1.5 \mathrm{mmol} / \mathrm{L})$; and $62 / 175$ (35.4\%) of patients 
Figure 1. LDL-C value according to calculated cardiovascular risk level (calculated using SCORE) at enrollment before (A) and after (B) starting on lipid-lowering therapy. LDL-C: low-density lipoprotein cholesterol; SCORE: Systemic Coronary Risk Estimation.

A

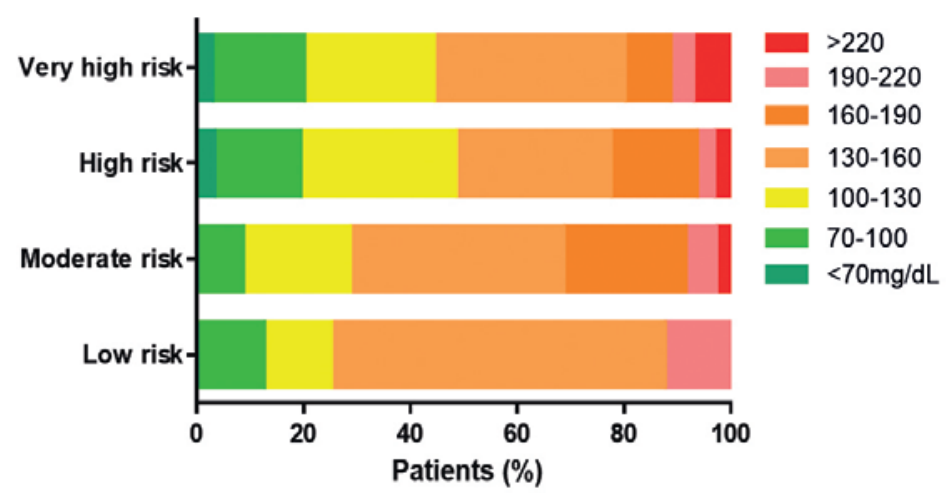

B

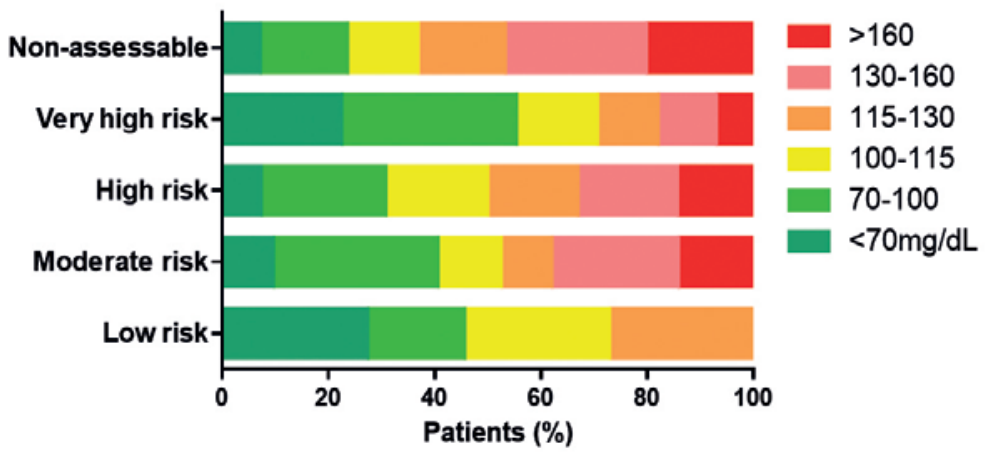

had an LDL-C value $>3.4 \mathrm{mmol} / \mathrm{L}(130 \mathrm{mg} / \mathrm{dL})$. The distributions of LDL-C levels according to CV risk levels are presented in figure 2. Mean high-density lipoprotein-cholesterol (HDL-C) concentration was 44.9 $\pm 15.3 \mathrm{mg} / \mathrm{dL}$. The corresponding value for triglycerides was $254 \pm 333 \mathrm{mg} / \mathrm{dL}$. The prevalence of mixed dyslipidemia was $60.6 \%$.

\section{Effect of LLT and LDL-C Goals}

At study enrollment, $97.4 \%$ were receiving a statin (62.8\% on statin monotherapy, $26.7 \%$ statin+fibrate, and $5.6 \%$ statin+ezetimibe (Table 1 ). About $28 \%$ of statin-treated patients were receiving high-intensity statin therapy (atorvastatin $40 / 80 \mathrm{mg}$ or rosuvastatin $20 / 40 \mathrm{mg}$ ), and $11.3 \%$ were on the highest dose regimen available in Mexico. Overall, patients had a median LDL-C decrease of $-23.1 \%(-43.7--2.4 \%)$ from diagnosis to the inclusion in the study. Close to half (58\%) of the cases had LDL-C change lower than $30 \%$; a $30-50 \%$ reduction occurred in $24 \%$; and a change greater than $50 \%$ occurred in merely $17.7 \%$ of the participants. Only $33 \%$ of the cases that had a
$50 \%$ LDL-C change had a high or very high rişk. On the other hand, only 240 cases (38.4\%) hadilDL-C below $100 \mathrm{mg} / \mathrm{dL}$ (the LDL-C goal accepted in the majority of the lipid guidelines). Furthermore, the goal of the intensive treatment $(<70 \mathrm{mg} / \mathrm{dL})$ was found only in 80 cases (12.8\%); $80 \%$ of them had bigh or very high CV risk. Other secondary lipid goals were not met in the study subjects. More than half (58.5\%) had $\mathrm{HDL}$ cholesterol below target value $(<40 \mathrm{mg} / \mathrm{dL}$ in men and $<50 \mathrm{mg} / \mathrm{dL}$ in women); this rate was not affected by the CV risk stratification. The sametrend was observed in the triglyceride concentrations, as triglyceride concentrations $>150 \mathrm{mg} / \mathrm{dL}$ were fônd in $52.2 \%$ of the participants.

\section{LDL-C Goal Achievement and CV Risk}

Patients at low CV risk had the highest percentage reduction of LDL-C followed by those at very-high-risk and moderate risk. Most patients did not achieve their target $\mathrm{LDL}-\mathrm{C}$ reduction according to the expected CV risk category (Table 2 ). The proportion of patients who achieved the LDL-C targets was higher in the 
Figure 2. Concordance between estimated cardiovascular (CV) risk by attending physicians compared to calculated CV risk using SCORE. Shaded areas represent over and underestimation of risk per calculated CV risk category. Weighted Kappa $=0.260$; 95\%Cl 0.206-0.314. SCORE: Systemic Coronary Risk Estimation.

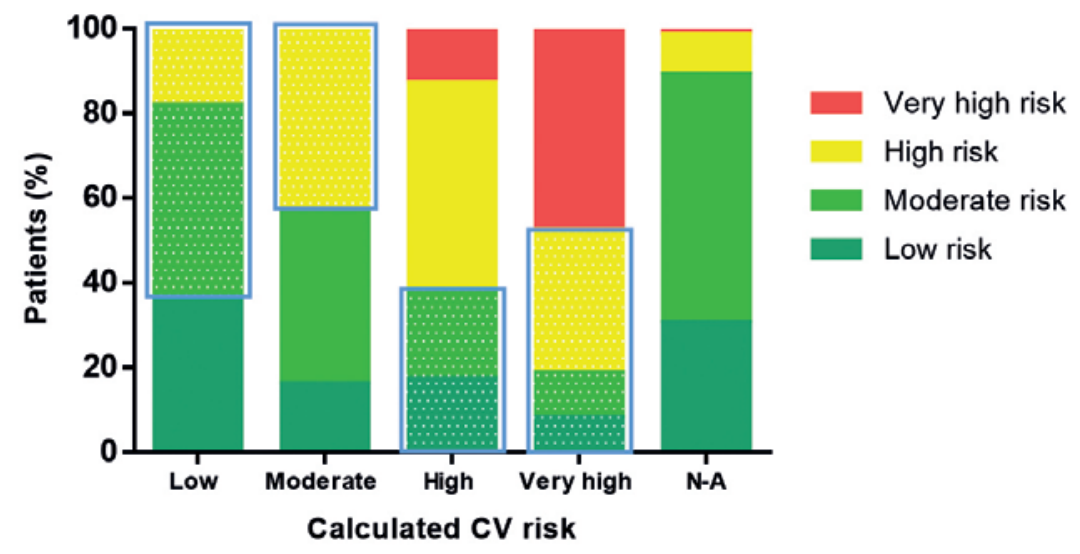

Table 1. Use of lipid-lowering therapies (LTT) by patients from the evaluated physicians, overall, and stratified according to cardiovascular risk level as evaluated by EUROSCORE

\begin{tabular}{|c|c|c|c|c|c|c|c|}
\hline Lipid-lowering therapy & $\begin{array}{c}\text { Total } \\
(\mathrm{n}=626)(\%)\end{array}$ & $\begin{array}{c}\text { Low risk } \\
(n=11)(\%)\end{array}$ & $\begin{array}{l}\text { Moderate risk } \\
(n=42)(\%)\end{array}$ & $\begin{array}{c}\text { High risk } \\
(n=235)(\%)\end{array}$ & $\begin{array}{l}\text { Very-high risk } \\
(\mathrm{n}=210)(\%)\end{array}$ & $\begin{array}{l}\text { Not as } \\
(n=1\end{array}$ & $\begin{array}{l}\text { ssesssable } \\
128)(\%)\end{array}$ \\
\hline Any statin & $610(97.4)$ & $9(81.8)$ & $42(100.0)$ & $227(96.6)$ & $208(99.0)$ & 124 & $(96.4)$ \\
\hline High-statin dosage & $173(28.4)$ & $4(44.4)$ & $12(28.6)$ & $62(27.3)$ & $70(33.7)$ & 25 & $(20.2)$ \\
\hline On highest statin dose & $69(11.3)$ & $1(11.1)$ & $5(11.9)$ & $16(7.0)$ & $36(17.3)$ & 11 & $(8.9)$ \\
\hline Statin monotherapy & $393(62.8)$ & $3(27.3)$ & $23(54.8)$ & $148(63.0)$ & $129(61.4)$ & 90 & $(7 \underline{0} .3)$ \\
\hline Statin+fibrate+other LLT & $165(26.3)$ & $6(54.6)$ & $11(26.2)$ & $66(28.1)$ & $62(29.6)$ & 20 & $(157)$ \\
\hline $\begin{array}{l}\text { Statin+cholesterol } \\
\text { absorption } \\
\text { inhibitor+other LLT }\end{array}$ & $35(5.6)$ & $0(0.0)$ & $3(7.1)$ & $11(4.7)$ & $12(5.7)$ & 10 & 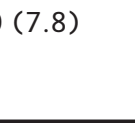 \\
\hline
\end{tabular}

moderate risk group and the lowest in the very-highrisk group (24.8\% for very high risk, vs. $26.4 \%$ for high-risk and $52.4 \%$ for moderate risk, Fig. 3). Among patients at high and very high CV risk, who were those with suboptimal LDL-C goals, $54.5 \%$ and $68.6 \%$, respectively, were either retired or unemployed and 13 patients at those $\mathrm{CV}$ risk levels had work disability due to $\mathrm{CV}$ disease, suggesting a potential financial reason contributing to $L D L-C$ goal non-achievement. The percentage of patients who achieved the target goals according to CV risk category when estimated by physicians was $35.9 \%$ versus $26.3 \%$ when $C V$ risk was assessed based on the ESC/EAS recommendations ( $p<0.001$ ). The concordance between physician-estimated $C V$ risk and guideline-estimated risk was moderate to strong $(\kappa=0.721,95 \% \mathrm{Cl} 0.652-$ 0.791 ), indicating that goal non-achievement could also stem from inadequate CV risk calculation.
Factors Associated with Non-Achievement of LDL-C Goals in the Study Sample

Patients at high or very high CV risk were approximately three-fold less likely to achieve their $\mathrm{LDL}-\mathrm{C}$ goals compared to patients at moderate $\mathrm{CV}$ risk ( $\mathrm{Ta}$ ble 2). Overweight and obesity, abdominal obesity, and metabolic syndrome defined using International Diabetes Federation criteria, statin intolerance,female sex, treatment in public versus private settings-diabetes, and hypertriglyceridemia were also associated with failure to achieve targets, whereas higher levels of education were associated with a lower risk of nonachievement of LDL-C goal. In relation to the etiology of dyslipidemia, we identified 426 patients with secondary dyslipidemia among whom only 110 (38.4\%) reached LDL-C goals. A similar scenario was observed 
Figure 3. Number of patients who achieved LDL-C goals according to the guideline used for lipid management (A), and to the cardiovascular risk calculated by SCORE (B). LDL-C: low-density lipoprotein cholesterol; SCORE: Systematic Coronary Risk Estimation.

A

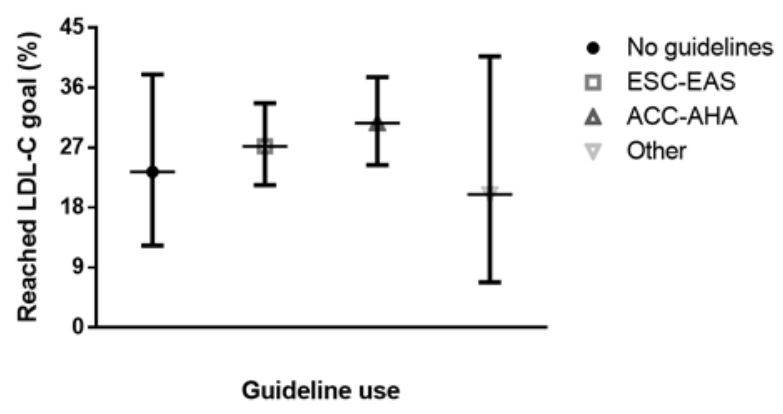

B

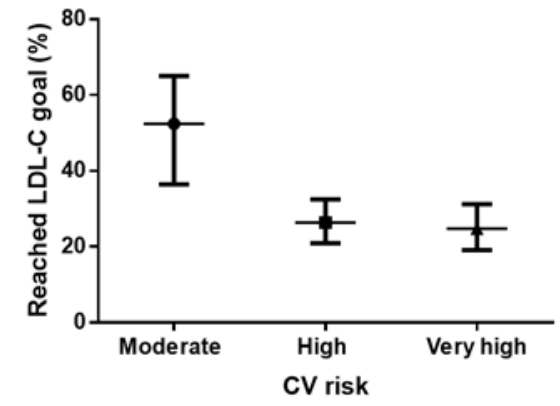

Table 2. Factors associated with decreased likelihood to achieve LDL-C goals according to ESC/EAS guidelines under statin therapy in Mexican population

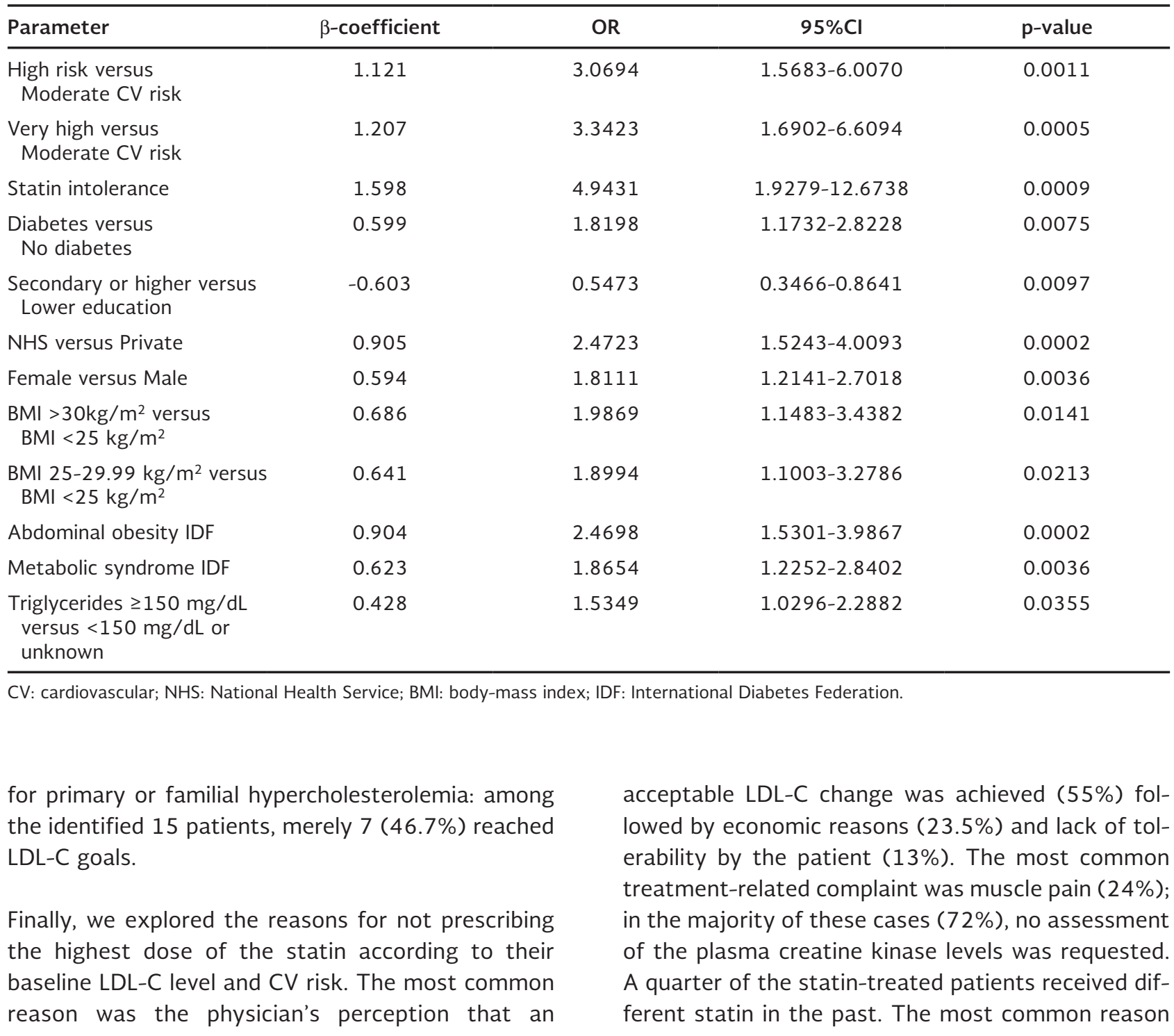


for changing the statin was the physician's decision based on the LDL-C achieved (70\%) followed by the cost of treatment (11.1\%).

\section{DISCUSSION}

ICLPS provides a real-life assessment of the effectiveness of the LLT in non-European countries. Here, we described the results of Mexican participants. Our results reveal major gaps in the implementation of stable LLT in a representative set of patients treated by experienced physicians; however, this issue is not limited to Mexico given that the same problem was observed in all countries included in ICLPS ${ }^{8}$. The percentage of cases that attained the LDL-C treatment goals was low, especially among very high and high CV-risk groups. As reported by the ICLPS study, only a quarter of very high and high CV-risk patients achieved their risk-based LDL-C targets as recommended by current guidelines. Our data identify areas of opportunity to improve effectiveness of the LLT prescription and CV risk estimation in practice. Guidelines should consider the inclusion of actions to avoid clinical inertia and identify the cases with the highest likelihood of abandoning LLT or being undertreated $^{11-14}$.

The ICPLS methodology explores both patient-related and physician-related factors that may limit the impact of LLT. Our results reveal significant disparities in LLT and LDL-C goal achievement, which impacts adequate $\mathrm{CV}$ risk management, treatment access, and might influence the effectiveness of LLT. Previous reports consistently showed lower LDL-C goal achievement rates in women, associated with the physician's perception of lesser CV risk and a higher probability to discontinue therapy ${ }^{13-15}$. Our results agree with the REGARDS study, which showed that older age, sex, race, poverty, and insurance type influence access to LLT and LDL-C goal achievement. Disparities in healthcare access in Mexico have also shown to decrease the likelihood of cholesterol screening, thus affecting disease identification and prompt initiation of treatment ${ }^{16-18}$. Data reported here may be useful for the Mexican health-care system to adapt approaches to reduce $\mathrm{CV}$-risk burden and decrease gaps limiting adherence, reducing intrinsic inequalities in the Mexican health-care system which restrain adequate management of $\mathrm{CV}$ risk.
The high prevalence of T2D is a peculiarity the Mexican population ${ }^{1,19}$. Data from ENSANUT 2006 reported that only $28.6 \%$ of individuals with $\mathrm{T} 2 \mathrm{D}$ had $\mathrm{LDL}-\mathrm{C}<100 \mathrm{mg} / \mathrm{dL}$ and $10.5 \%$ had LDL-C < $00 \mathrm{mg} /$ $\mathrm{dL}$ and projected that over two-thirds of patients with T2D in Mexico were not at ESC/EAS goal levèls but were nonetheless, eligible for LLT. Furthermore, ENSANUT 2012 showed that $<3 \%$ of patients with T2D were under statin therapy ${ }^{20}$. T2D increases the risk of hypertriglyceridemia, which leads to an underestimation of LDL-C using the Friedewald equation ${ }^{2}$. Recent data showed that LDL-C estimation using Martin's formula is more accurate in Mexican patients with familial combined hyperlipidemia, suggesting it may be a useful tool to address LDL-C undertreatment in patients with comorbid hypertriglyceride$\mathrm{mia}^{22}$. Furthermore, abdominal obesity, polypharmacy, and depression are diabetes-related comorbid conditions, which may also interfere with the adherence to LLT. These comorbidities interfere with $L D L-C$ management in T2D and might explain the adverse association observed in relation to goal non-achievement in T2D in our cohort, contrasting with data from ICLPS.

LDL-C goal non-achievement is reliant both oraccurate $\mathrm{CV}$ risk estimation and the proper selection of statin dosage by the practitioner. In our study.pphysician-estimated risk influenced statin treatmeneintensification, indicating the necessity to improve EV risk estimation with population-specific data, such as those provided by the Globorisk collaboration23. Our data strongly suggest that clinical inertia and nonadherence to guidelines are common and should be considered a target for public health policies 24,25 . Increasing LLT adherence thus relies on overcoming many factors which affect access to treatment and treatment adherence itself; therefore, it is possible that the therapeutic gap for goal non-achievement in very-high and high CV risk patients may not be fully mitigated by use of statin therapy alone and that local guidelines should work on improving recommendations related to treatment assignment and targeted treatment with more effective LLTs. Complementary actions to be considered are combination therapies with ezetimibe and/or PCSK9 inhibitors which have shown that decreasing LDL-C to lower levels than recommended by guidelines may provide additional CV benefit ${ }^{26-28}$. Studies of LLT combinations $\frac{1}{4}$ T2D and metabolic syndrome may shed light on the added 
benefits of such therapies in settings like Mexico, where hypercholesterolemia often is the result of comorbid metabolic abnormalities.

Our study had some strengths and limitations. The observational setting provides real-world evidence for benefits of LLT and the barriers of LDL-C goal achievement in everyday clinical practice; nevertheless, it is subject to limitations including lack of data before clinical diagnosis of primary and secondary dyslipidemias, treatment-dosage specifications, and difficulty in assigning causality to our observations. Even though random selection of centers and physicians reduced selection bias, most of our patients were from urban settings, and a large proportion had non-public insurance, which limits extrapolation of our results to a national scale with underrepresentation of non-urban or public-sector settings. Furthermore, the use of SCORE may affect CV risk estimation in our population, where it has been shown to offer questionable risk prediction ${ }^{29}$. Finally, we did not assess the role of apolipoprotein B as an LLT goal to reduce CV risk; given the role of apolipoprotein $\mathrm{B}$ in the pathogenesis of atherosclerosis and CVD, this remains an area of opportunity for future studies.

The achievement of LDL-C goals in Mexico is suboptimal and even lower compared to other countries included in the ICLPS collaboration. Regional differences related to intrinsic metabolic burden, health care, and social determinants of health intervene in proper LDL-C goal achievements and suggest that LLT should be tailored to meet necessities of individual countries. These interventions should be in attendance to country-specific disparities and considering intrinsic risk for conditions which alter atherogenic profiles, including diabetes, metabolic syndrome, and insulin resistance. The development of local guidelines which aim to reduce barriers for LLT access, optimize treatment intensity assignment and use of combination therapies should result in increased goal achievement and a substantial decrease in CV disease rates in Mexico.

\section{ACKNOWLEDGMENTS}

This research was conducted as part of the International ChoLesterol management Practice Study (ICLPS). This project was sponsored by Sanofi. Omar Yaxmehen
Bello-Chavolla is enrolled at the Plan of Combined Studies in Medicine MD/PhD (PECEM) program at the Faculty of Medicine at the National Autonomous University of Mexico (UNAM) and is supported by CONAACYT.

This study was sponsored by Sanofi, Mexico. The funder had no role in data collection, analysis of the results or preparation of the manuscript.

\section{REFERENCES}

1. Aguilar-Salinas CA, Gómez-Pérez FJ, Rull J, Villalpando' $\bar{S}$, Barquera S, Rojas R, et al. Prevalence of dyslipidemias in the Mexican national health and nutrition survey 2006. Salud Publica Mex. 2010;52 Suppl 1:S44-53.

2. Cholesterol Treatment Trialists' (CTT) Collaboration, Baigent C, Blackwell L, Emberson J, Holland LE, Reith C, et al. Efficacy and safety of more intensive lowering of LDL cholesterol:a metaanalysis of data from 170,000 participants in 26 randomised trials. Lancet 2010;376:1670-81.

3. Stone NJ, Robinson JG, Lichtenstein AH, Bairey Merz CN, Blum $\mathrm{CB}$, Eckel RH, et al 2013 ACC/AHA guideline on the treatment of blood cholesterol to reduce atherosclerotic cardiovascular risk in adults: a report of the American college of cardiology/ American heart association task force on practice guidelines. J Am Coll Cardiol. 2014;63:2889-934

4. Jacobson TA, Ito MK, Maki KC, Orringer CE, Bays HE, Jones PH, et al. National lipid association recommendations for-patientcentered management of dyslipidemia: part 1 executive summary. J Clin Lipidol. 2014;8:473-88.

5. Piepoli MF, Hoes AW, Agewall S, Albus C, Brotons C, "Gatapano $A L$, et al. 2016 European guidelines on cardiovascular disease prevention in clinical practice: the sixth joint task force of the European society of cardiology and other societies on cardiovascular disease prevention in clinical practice (constituted by representatives of 10 societies and by invited experts): developed with the special contribution of the European association for cardiovascular prevention and rehabilitation (EACPR). Eur J Prev Cardiol. 2016;23:NP1-96.

6. Reiner Ž, De Backer G, Fras Z, Kotseva K, Tokgözoglud, Wood $D$, et al. Lipid lowering drug therapy in patients with coronary heart disease from 24 European countries Findings from the EUROASPIRE IV survey. Atherosclerosis. 2016;246:243-50.

7. Shioji K, Izuhara M, Mitsuoka H, Uegaito T, Matsuda M, Kishiwada Atherosclerosis Prevention Study (KAPS) Group. et al. Achievement rates of japan atherosclerosis society gtidelines 2007 LDL-cholesterol goals with rosuvastatin or atorvastatin in patients who had not achieved their goal with atovastatin. Cardiovasc Ther. 2014;32:97-104.

8. Danchin N, Almahmeed W, Al-Rasadi K, Azuri J, Berrah A, Cuneo CA, et al. Achievement of low-density lipoprotein cholesterol goals in 18 countries outside Western Europe: the international choLesterol management practice study (ICLPS). Eur J Prev Cardiol. 2018;25:1087-94.

9. Conroy RM, Pyörälä K, Fitzgerald AP, Sans S, Menotti A, De Backer $G$, et al. Estimation of ten-year risk of fatal cardiovascular disease in Europe: the SCORE project. Eur Heart ${ }^{+1}$. 2003; 24:987-1003.

10. Austin MA, Hutter CM, Zimmern RL, Humphries SE Genetic causes of monogenic heterozygous familial hypercholesterolemia: a HuGE prevalence review. Am J Epidemioh 2004; 160:407-20

11. Waters DD, Brotons C, Chiang CW, Ferrières J, Foody 'J, Jukema JW, et al. Lipid treatment assessment project 2: a multinational survey to evaluate the proportion of patients achieving low-density lipoprotein cholesterol goals. Circulation. 2009; 120:28-34

12. Pearson TA, Laurora I, Chu H, Kafonek S. The lipid treatment assessment project (L-TAP): a multicenter survey to evaluate the percentages of dyslipidemic patients receiving lipid-lowering therapy and achieving low-density lipoprotein cholesterol goals. Arch Intern Med. 2000;160:459-67. 
13. Russo G, Pintaudi B, Giorda C, Lucisano G, Nicolucci A, Cristofaro MR, et al. Age and gender-related differences in LDL-cholesterol management in outpatients with Type 2 diabetes mellitus. Int J Endocrinol. 2015;2015:957105.

14. Rodriguez F, Olufade TO, Ramey DR, Friedman HS, Navaratnam $P$, Heithoff $K$, et al. Gender disparities in lipid-lowering therapy in cardiovascular disease: insights from a managed care population. J Womens Health (Larchmt). 2016;25:697-706.

15. Zhang X, Ji L, Ran X, Su B, Ji Q, Hu D, et al. Gender disparities in lipid goal attainment among Type 2 diabetes outpatients with coronary heart disease: results from the CCMR-3B study. Sci Rep. 2017;7:12648.

16. Schroff P, Gamboa CM, Durant RW, Oikeh A, Richman JS, Safford $M M$, et al. Vulnerabilities to health disparities and statin use in the REGARDS (Reasons for geographic and racial differences in stroke) study. J Am Heart Assoc. 2017;6:5449.

17. Rivera-Hernandez M, Galarraga O. Type of insurance and use of preventive health services among older adults in Mexico. J Aging Health. 2015;27:962-82.

18. Gakidou E, Mallinger L, Abbott-Klafter J, Guerrero R, Villalpando $\mathrm{S}$, Ridaura RL, et al. Management of diabetes and associated cardiovascular risk factors in seven countries: a comparison of data from national health examination surveys. Bull World Health Organ. 2011;89:172-83.

19. Bello-Chavolla OY, Rojas-Martinez R, Aguilar-Salinas CA, Hernández-Avila M. Epidemiology of diabetes mellitus in Mexico. Nutr Rev. 2017;75:4-12.

20. Gómez-Pérez FJ, Rojas R, Villalpando S, Barquera S, Rull ], Aguilar-Salinas CA, et al. Prevention of cardiovascular disease based on lipid lowering treatment: a challenge for the Mexican health system. Salud Publica Mex. 2010;52 Suppl 1:S54-62.

21. Nichols GA, Philip S, Reynolds K, Granowitz CB, O'Keeffe-Rosetti $M$, Fazio $S$, et al. Comparison of medical care utilization and costs among patients with statin-controlled low-densitylipoprotein cholesterol with versus without hypertriglyceridemia. Am J Cardiol. 2018;122:1128-32.

22. Mehta R, Reyes-Rodríguez E, Yaxmehen Bello-ChavollaO Guerrero-Díaz AC, Vargas-Vázquez A, Cruz-Bautista I, et al. Performance of LDL-C calculated with martin's formula compared to the friedewald equation in familial combined hyperlipidemia. Atherosclerosis. 2018;277:204-10.

23. Hajifathalian K, Ueda P, Lu Y, Woodward M, Ahmadivand A, Aguilar-Salinas CA, et al. A novel risk score to predict cardiovascular disease risk in national populations (Globorisk); 'a pooled analysis of prospective cohorts and health examination-surveys. Lancet Diabetes Endocrinol. 2015;3:339-55.

24. Vonbank A, Drexel H, Agewall S, Lewis BS, Dopheide JF, Kjeldsen $\mathrm{K}$, et al. Reasons for disparity in statin adherence ratesbetween clinical trials and real-world observations: a review. Eur Heart ] Cardiovasc Pharmacother. 2018;4:230-6.

25. De Vera MA, Bhole V, Burns LC, Lacaille D. Impact of statin adherence on cardiovascular disease and mortality outcomes: a systematic review. Br J Clin Pharmacol. 2014;78:684-98.

26. Cannon $C P$, Blazing MA, Giugliano RP, McCagg $A$ White JA, Theroux $P$, et al. Ezetimibe added to statin therapy after acute coronary syndromes. N Engl J Med. 2015;372:2387-97.

27. Roth EM. Alirocumab for hyperlipidemia: ODYSSEY phase III clinical trial results and US FDA approval indications. Future Cardiol. 2016;12:115-28.

28. Schwartz GG, Steg PG, Szarek M, Bhatt DL, Bittner VA, Diaz R, et al. Alirocumab and cardiovascular outcomes after acute coronary syndrome. N Engl J Med. 2018;379:2097-107.

29. Rodríguez-Chávez LL, Figueroa-Solano J, Muñoz-Consuegra CE, Avila-Vanzzini N, Kuri-Alfaro J. EuroSCORE underestimate the mortality risk in cardiac valve surgery of Mexican population. Arch Cardiol Mex. 2017;87:18-25. 